\title{
Las infraestructuras aeroportuarias: tipo de propiedad y su relación con la eficiencia*
}

\author{
Claudia María Giraldo-Velásquez ${ }^{*}$ \\ Amanda Stella Valderrama Castañeda ${ }^{* * *}$ \\ Sandra Zapata-Aguirre
}

Recibido: 07/08/2014 • Aceptado: 15/04/2015

\begin{abstract}
Resumen
El transporte aéreo es un sector caracterizado por ser uno de los de más rápido crecimiento en la economía mundial. Este aumento explosivo constituye un desafío para los aeropuertos en términos de desarrollo de la capacidad y la gestión. La conversión hacia la comercialización y privatización de la infraestructura aeroportuaria ha seguido los procesos de desregulación y privatización de las aerolíneas, aunque con un importante retraso. Entre los motivos que han conducido a estos procesos se encuentra la necesidad de mejorar su eficiencia para enfrentar una industria aeronáutica altamente competitiva. Este artículo de reflexión tiene como punto de partida la idea, generalmente aceptada, de que la privatización como modelo de propiedad o gestión está asociada con una mayor productividad. Los trabajos de referencia abordados hacen mención a aquellos cuyo análisis de la eficiencia es hecho a través de la técnica DEA por ser una de las más consolidadas y aceptadas en la literatura.
\end{abstract}

Palabras clave: aeropuertos, infraestructuras aeroportuarias, transporte aéreo, eficiencia, tipos de propiedad

\footnotetext{
" Este artículo de reflexión hace parte de los productos obtenidos del proyecto "Análisis de las infraestructuras aeroportuarias como determinantes de la competitividad de un destino turístico” financiado por la Institución Universitaria Colegio Mayor de Antioquia, a quien las autoras agradecen.

** Profesora investigadora de la Facultad de Administración. Directora del Grupo de Investigación Empresarial y de Turismo-GIET (categoría B-Colciencias). Institución Universitaria Colegio Mayor de Antioquia. Carrera 78 \# 65-46; teléfono: +574 4445611 ext. 147. Email: cgiraldo@colmayor.edu.co

*** Profesora y Coordinadora Académica de la Facultad de Administración. Institución Universitaria Colegio Mayor de Antioquia. Carrera 78 \# 65-46; teléfono: +574 4445611 ext. 147. Email: svalderrama@colmayor.edu.co

**** Investigadora adscrita al Grupo de investigación GIET. Grupo de Investigación Empresarial y de Turismo-GIET (categoría B-Colciencias). Institución Universitaria Colegio Mayor de Antioquia. Carrera 78 \# 65-46; teléfono: +574 4445611 ext. 147 Email: sandra.zapata@colmayor.edu.co

Autor para correspondencia.
}

Revista Ingenierías Universidad de Medellín, vol. 14, No. 27 pp. 179-194 ISSN 1692-3324 - julio-diciembre de 2015/294 p. Medellín, Colombia 


\section{Airport infrastructures: forms of ownership and the relationship with efficiency}

\section{Abstract}

Air transport has been characterized for being one of the fastest growing sectors. This significant growth is a challenge for airport infrastructures in terms of capacity and management. The shift to commercialization and privatization of airports has followed the same path of the deregulation and privatization of airlines, but with certain delayed. One of the common reasons for these new processes is the improvement in efficiency levels in order to face a highly competitive industry. This reflection paper focuses on a generalized idea that considers the form of a firm ownership and its management model is positively associated to high levels of productivity. Studies addressed in the present paper are limited to those papers considering DEA as the technique of efficiency analysis for being one of the most accepted in the academic literature.

Key words: airports, airport infrastructures, air transport, efficiency, types of ownership. 


\section{INTRODUCCIÓN}

La industria del transporte aéreo facilita la mayor parte de los viajes, las relaciones económicas y el comercio exterior mundiales, haciendo posible conectar un país con la economía global. Asimismo, ayuda a mejorar los niveles de productividad para el beneficio de una economía [1] . Para algunos países el transporte aéreo es un sector estratégico que incentiva el empleo y el crecimiento económico de largo plazo a través de varios canales directos, indirectos, inducidos y catalizadores. Algunos de estos incluyen la actividad generada por el gasto de los visitantes extranjeros que viajan por el territorio nacional vía aérea y el nivel de comercio facilitado directamente por el transporte de mercancías de acuerdo con los reportes elaborados por la Asociación Internacional de Transporte Aéreo (IATA, por sus siglas en inglés).

Una parte esencial dentro del sistema del transporte aéreo está representada por los aeropuertos, que proveen toda la infraestructura necesaria para hacer posible que pasajeros y carga sean transferidos de la superficie, hasta los distintos modos aéreos de transporte y que las líneas aéreas puedan realizar sus despegues y aterrizajes. La infraestructura básica aeroportuaria está formada por las pistas, las pistas de carreteo, la zona de aparcamiento de los aviones, los puentes de embarque, las terminales de pasajeros y de carga y los intercambios de transporte en tierra.

Actualmente, el transporte aéreo es un sector caracterizado por ser uno de los de más rápido crecimiento en la economía mundial. Este aumento explosivo constituye un desafío para los aeropuertos en términos de desarrollo de la capacidad y la gestión. Asimismo, el sector aeroportuario no es el mismo de hace unas décadas, cuando las infraestructuras aeroportuarias operaban como cualquier otro servicio público. Ahora se trata de un sector dinámico y competitivo, y las empresas que lo forman han comenzado a explotar su faceta comercial y, por tanto, a operar como un negocio con la obligación de generar ingresos. Es decir, a operar de manera más eficiente.

La conversión hacia la comercialización y privatización de la infraestructura aeroportuaria ha seguido los procesos de desregulación y privatización de las aerolíneas, aunque con un importante retraso. Entre los motivos que llevan al cambio de gobernanza de los aeropuertos se encuentran desde la búsqueda de fondos para la expansión y la renovación de la capacidad aeroportuaria, hasta la necesidad de mejorar su eficiencia para enfrentar una industria aeronáutica altamente competitiva [2].

Existe la idea, generalmente aceptada, de que la privatización está asociada con una mayor productividad, y por tal razón en la literatura académica se ha producido una serie de estudios que buscan evaluar el impacto del tipo de propiedad y las consecuencias de la privatización sobre la eficiencia [2]. 
Las medidas de eficiencia han sido ampliamente conocidas por los gestores $\mathrm{u}$ operadores aeroportuarios quienes son conscientes de su importancia como una guía adecuada en la planificación estratégica y en el análisis de la posición competitiva de estas organizaciones en la industria aeroportuaria [3], lo que hace que tengan grandes presiones para investigar el desempeño de sus competidores utilizando herramientas de benchmarking [4], [5], [6].

La proliferación de estudios empíricos que analizan la eficiencia de infraestructuras aeroportuarias confirma que al día de hoy es uno de los temas relevantes en la investigación específica de la economía y la gestión aeroportuaria.

Considerando que han pasado ya más de tres décadas desde la ola de procesos de privatización de las infraestructuras aeroportuarias en el mundo, el objetivo del presente trabajo es hacer un análisis de lo que se entiende por eficiencia en el sector del transporte aéreo, cómo suele medirse y, finalmente, comprender hasta qué punto la privatización ha contribuido a un mejor desempeño de las mencionadas infraestructuras. Se espera llamar la atención sobre esta temática que, para el caso de Colombia y los países de la región, ha sido escasamente abordada. La reflexión de este trabajo tiene como punto de partida la idea, generalmente aceptada, de que la privatización como modelo de propiedad o gestión está asociada con una mayor productividad. En el caso concreto de las infraestructuras aeroportuarias, los trabajos de referencia abordados hacen mención a aquellos cuyo análisis de la eficiencia es realizado a través de la técnica DEA por ser una de las más consolidadas, aceptadas y ampliamente utilizadas en los estudios internacionales de eficiencia económica. En las figuras 1 y 2 se puede apreciar una lista de los trabajos más recientes de la literatura. Con esta técnica aparecen estudios de eficiencia para aeropuertos europeos, los cuales han sido trabajados en la escala de país, destacándose por su cantidad, los trabajos para Italia y España [7], [8], [9]; de igual manera, han sido analizados en conjunto dichos aeropuertos europeos [10], [11]. Para Asia se encuentran estudios principalmente con datos de aeropuertos de China [12], [13], [14], [15], [16].

Los estudios con la aplicación del método DEA para el caso latinoamericano son escasos [17], [18] [19] . Esto puede deberse a la falta de información pública. Sin embargo, como análisis de conjunto de aeropuertos, aparece un estudio en el que son analizados 21 aeropuertos en el período 2000-2007 [20]. 


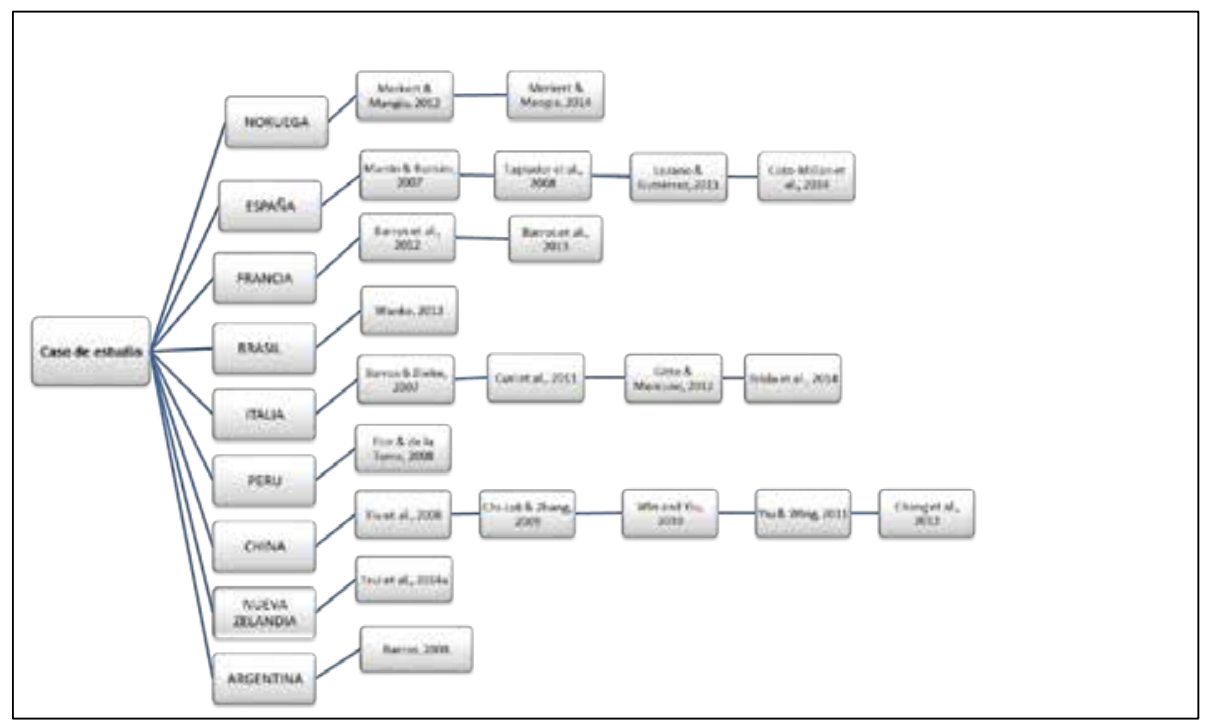

Figura 1. Estudios abordados en un nivel nacional Fuente: elaboración propia

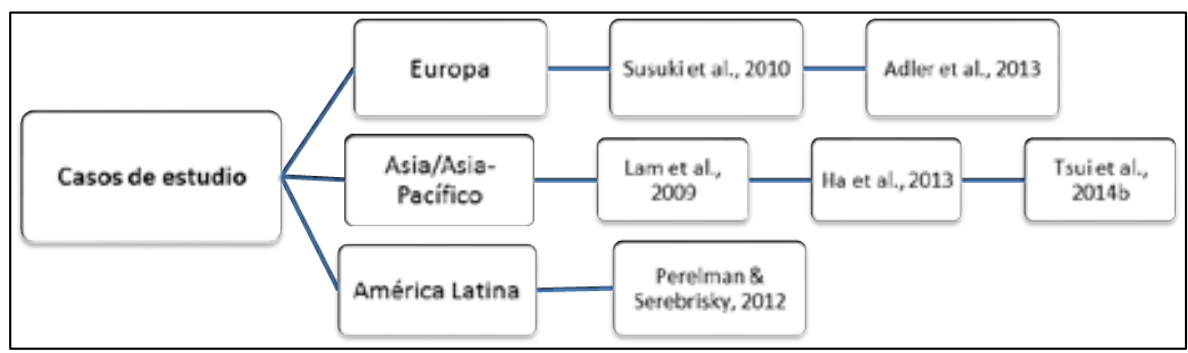

Figura 2. Estudios abordados en un nivel regional. Fuente: elaboración propia

\section{DESEMPEÑO ECONÓMICO: MIDIENDO LA EFICIENCIA}

La monitorización sistemática y la comparación del desempeño económico de las infraestructuras aeroportuarias frente a sus pares son prácticas que no se generalizaron sino recién a comienzos de los años 80 . De alguna forma este comportamiento lo explican las leves presiones comerciales y organizacionales hacia los aeropuertos, más la falta de experiencia en el uso de técnicas de benchmarking dentro del sector público en general. A partir del fenómeno de la comercialización y privatización de las infraestructuras aeroportuarias, el interés por dichas técnicas de gestión comienza a ganar interés. 
De manera gradual, las infraestructuras aeroportuarias fueron consideradas mucho más como empresas comerciales. Es así como actualmente se muestran como un sector dinámico y competitivo, y las empresas que lo forman han comenzado a explotar su faceta comercial y, por tanto, a funcionar como un negocio con la obligación de generar ingresos. Por tanto, se manejan con una orientación más comercial en la que los responsables de la operación aeroportuaria son proclives a indagar acerca de su competencia, quiénes son los mejores jugadores y cuáles ejecutan las mejores prácticas. Estos análisis de desempeño comparativo les permiten a los responsables tener un conocimiento profundo acerca de diversas cuestiones tales como si los aeropuertos privatizados operaran de manera más eficiente que aquellos gestionados por el ente público.

En dicho sentido ha surgido en la literatura una amplia gama de trabajos que dan cuentan de las diferentes metodologías de las que se puede valer el sector aeroportuario para medir su desempeño. Dichas medidas analizan la relación existente entre insumos y productos en un aeropuerto, relación que puede ser expresada tanto en términos físicos como financieros.

Como con otros negocios, el factor trabajo y el capital son los mayores inputs del sistema aeroportuario. La medida física más simple del insumo "trabajo" es el número total de trabajadores. Por su parte, determinar la medida más fiable del insumo "capital" es mucho más difícil. En términos físicos, el insumo capital es medido por la capacidad de producción o la capacidad del sistema. En el caso de un aeropuerto esto no puede evaluarse bajo una sola medida. La capacidad de las pistas, terminales, fajas transportadoras de equipaje, entre otros, debe ser considerada.

El concepto de eficiencia productiva se puede representar a través de una frontera de producción o una frontera de costo. La frontera de producción representa la máxima cantidad de producto obtenible con un determinado nivel de factores productivos, mientras que la frontera de costo representa la mínima cantidad de insumos con la cual se puede producir una cantidad determinada de producto orientada al incremento de su eficiencia.

A partir de una propuesta previa en la literatura, Charnes et al. [21] desarrollaron la metodología del Análisis Envolvente de Datos o DEA (siglas en inglés para Data Envelopment Analysis que luego sería ampliamente utilizada en la literatura) la cual sirve de apoyo a las decisiones basada en programación lineal para identificar funciones de producción empíricas, y que es usada para la evaluación de unidades relativas entre las que se encuentra el aeropuerto; esta técnica es una herramienta de evaluación comparativa (benchmarking) [22]. 
Los modelos DEA identifican:

- La eficiencia relativa para un conjunto de unidades comparables.

- Unidades de referencia para las unidades ineficientes.

- Una frontera de eficiencia desde el punto de vista de las mejores prácticas observadas.

Los modelos DEA existentes pueden bien estar orientados a los inputs o los outputs. Los primeros tienen como objetivo minimizar el nivel de entradas produciendo por lo menos el mismo nivel de salidas observadas. En tanto que los modelos orientados a los outputs tienen por objetivo maximizar el nivel de salidas manteniendo constante el nivel de entradas observadas.

Uno de los trabajos de mayor influencia [23] destaca las ventajas de esta metodología cuando se estudia la eficiencia de aeropuertos y se establece un modelo de gestión aeroportuario basado en dos productos: servicios del terminal (como el movimiento de pasajeros) y los movimientos de las aeronaves.

Para los objetivos del presente trabajo se han tomado como referencia exclusivamente los estudios que han analizado la eficiencia de los aeropuertos a través de la técnica DEA. La razón para ello se sustenta en el hecho que dicha metodología es una de las más consolidadas, aceptadas y ampliamente utilizadas internacionalmente en los estudios de eficiencia económica [24], lo cual le confiere la virtud de ser un instrumento útil para apoyar las acciones de los agentes encargados de la toma de decisiones en el sector de las infraestructuras aeroportuarias.

\section{LA PROPIEDAD Y LA GESTIÓN AEROPORTUARIA}

Históricamente los aeropuertos fueron de propiedad y operación gubernamental. Sin embargo, desde los años 80 se han presentado cambios importantes en la manera en que estas infraestructuras son gestionadas y operadas, al tiempo que ha cambiado su estructura de propiedad. Los motivos que han llevado a esta restructuración son diversos y van desde la búsqueda de un acceso más fácil a la obtención de fondos privados, hasta el mejoramiento de la eficiencia operacional.

En la actualidad los aeropuertos han ido saliendo del espacio de lo público para insertarse en el campo de lo privado, lo que se ha conocido como el fenómeno de la privatización. Esto ha surgido como producto de un cambio en el paradigma, que ha pasado de mirar el aeropuerto como un servicio público, hacia una mirada de este como una empresa de negocios para dar respuesta una industria del transporte aéreo más exigente, desregulada y competitiva [6]. 
Es necesario puntualizar que en lo relacionado con la privatización existen dos aspectos de gran importancia; la privatización de la gestión y la privatización de la propiedad de las instalaciones.

\section{TIPOS DE GESTIÓN}

El gestor, como su nombre lo indica, gestiona, supervisa y coordina el mantenimiento del aeropuerto, y puede ser el propietario o no [25]. Por tanto, puede haber privatización de la gestión aeroportuaria, pero manteniendo la propiedad pública de las instalaciones, como es el caso de los aeropuertos de Europa oriental. Otros países como Grecia, Bélgica (Bruselas), Dinamarca (Copenhague) y Austria (Viena) tienen los aeropuertos con gestión público- privada, y en Alemania e Italia se conserva la propiedad pública de todos los aeropuertos, pero hay varios tipos de gestión; pública, público- privada, y solo privada.

En la cadena de valor del transporte aéreo, aspectos como asistencia en tierra, minoristas, empresas de comidas, operadores de aparcamiento, mantenimiento de aviones, limpieza y seguridad en las áreas terminales han sido asumidos por las aerolíneas, o por la gerencia del aeropuerto, o se han creado compañías específicas para trabajar al respecto. Igualmente, los sistemas de control aéreo que tradicionalmente en todos los países han correspondido al manejo del sector público han sido privatizados.

Un ejemplo que vale la pena mencionar en lo relacionado con el reparto de la gestión aeroportuaria es el del aeropuerto de Zurich en Suiza, que tiene en cuenta tres áreas de gestión: la primera hace referencia a la técnica de control aéreo como el movimiento de aviones en pista, la segunda hace alusión a la logística del nodo de transporte aéreo en lo referente a las tasas aeroportuarias y la tercera tiene que ver con lo inmobiliario, como son los espacios comerciales, o hangares de mantenimiento, donde el aeropuerto y la gestión del transporte aéreo son públicos pero la explotación inmobiliaria está a cargo de una entidad mixta, con participación de capital privado.

También es posible hablar de una gestión integrada, en la cual los aeropuertos se gestionan en conjunto, donde existe una caja única que sirve para la financiación de aquellos aeropuertos que no sean rentables. Y los aeropuertos de gestión individualizada, que tienen autonomía financiera, y compiten con precios para la captación de compañías aéreas [25].

\section{TIPOS DE PROPIEDAD}

Por muchos años, los gobiernos fueron los propietarios y gestores de los aeropuertos; sin embargo, en las dos últimas décadas ha habido un cambio hacia la financiación del sector privado a través de la privatización parcial o total de los aeropuertos. 
Las distintas formas de estructuras de propiedad de los aeropuertos se pueden clasificar en las siguientes categorías: las de propiedad y operación por parte del gobierno, las de propiedad del gobierno pero de operación privada (parcialmente privada) y los totalmente privados [2].

a) La forma de propiedad y operación gubernamental está orientada principalmente hacia la función primaria del aeropuerto sin atender otras fuentes de valor comercial para la gestión aeroportuaria. Lo que suele presentarse aquí es que los aeropuertos compiten por inversión con otras prioridades gubernamentales. Algo que también suele presentarse es que este tipo de aeropuertos no tienen una visión de largo plazo respecto a las inversiones en infraestructuras. Ejemplos de este tipo de estructura se encuentran en Chile, Finlandia, Singapur, España o Suecia.

b) En muchos casos, los gobiernos han optado por la privatización parcial para, de esta forma, conseguir imponer algún tipo de regulación de precios. Sin embargo, aún existen gobiernos que se resisten a renunciar a la propiedad o el control público [2]. Estos aeropuertos tienen un objetivo comercial con fines de lucro, y aunque el gobierno sigue siendo el accionista mayoritario, siguen siendo capaces de tomar decisiones y desarrollar estrategias que un aeropuerto estatal no podría hacer.

c) En la literatura consultada pudo observarse que en lo relacionado con la privatización, el porcentaje de aeropuertos con una privatización total es pequeño [6]. En países como Australia, Nueva Zelanda e Inglaterra, los aeropuertos son totalmente privados y operan en una forma completamente diferente de la de los aeropuertos de propiedad pública en cualquier parte. Estos suelen tener una fuerte orientación hacia el mercado y hacia el cliente.

Según [26], citado en [25], existen tres tipos de concesiones aeroportuarias: las de apoyo donde se presta servicio en tierra a las aerolíneas, las comerciales que incluyen tiendas y aparcamientos, y las industriales que se encuentran en el ámbito aeroportuario por la cercanía. También, se presentan casos en los cuales una concesión ha pasado a ser un contrato de gestión, donde un grupo administra en nombre de la autoridad. En lo concerniente a la infraestructura para desarrollar obras civiles se han creado los contratos de obras públicas, en los cuales el sector privado operará, gestionará, desarrollará y financiará dicha infraestructura, pero el sector público en algún momento podrá vender el activo [27].

En conclusión, pese a la falta de una definición uniforme de las opciones de gobernabilidad, todas las clasificaciones destacan el grado de control gubernamental que existe, el cual es por lo general un elemento clave que influye en la elección de tipo de modelo a elegir por parte de los entes públicos [6]. 


\section{MODELO DE PROPIEDAD Y GESTIÓN Y SU EFECTO SOBRE LA EFICIENCIA}

Los efectos del tipo de propiedad de las infraestructuras aeroportuarias sobre la eficiencia productiva han sido tema de investigación importante tanto en la literatura económica como en la de administración. Esta misma sugiere que el tipo de propiedad afecta el desempeño de las organizaciones ya que cada propietario busca objetivos distintos y posee incentivos diversos.

Los tipos de propiedad son importantes dado que permiten identificar el efecto de estos sobre la eficiencia. Es así como las mejoras en los niveles de eficiencia son unos de los objetivos o razones por las que se opta por la privatización [1,5].

La mayoría de los estudios que analizan esta relación se han diseminado en los últimos años, particularmente entre 2006 y 2013, lo cual refleja no solo el creciente interés en la privatización sino también en los estudios de eficiencia [6] .

Como se ha indicado en la introducción, los estudios a los que se hace referencia en este trabajo son aquellos que utilizan como técnica de evaluación el DEA, pues existe una literatura que aborda otras metodologías. A continuación, se contrastan los principales resultados obtenidos en literatura académica, que utilizando la metodología DEA, analizan los efectos del tipo de propiedad sobre la eficiencia y son consignados en la tabla 1. Como se evidencia, los resultados son mixtos y, en cierta forma, no concluyentes; es decir, no hay un consenso claro de sus efectos.

Tabla 1. Principales resultados en el análisis de los efectos del tipo de propiedad y el desempeño de la eficiencia en los estudios que utilizan la técnica DEA

\begin{tabular}{|l|l|l|l|}
\hline \multicolumn{1}{|c|}{ Artículo } & \multicolumn{1}{|c|}{ Principal conclusión } & \multicolumn{1}{c|}{ Caso de estudio } & \multicolumn{1}{c|}{ Método } \\
\hline Parker, 1999 & La privatización no tiene efecto sobre la eficiencia & $\begin{array}{l}\text { Reino Unido } \\
\text { (BAA) }\end{array}$ & DEA \\
\hline Oum, 2003 & $\begin{array}{l}\text { No hay una relación significativa del tipo de propiedad } \\
\text { y las eficiencias operacional y financiera }\end{array}$ & $\begin{array}{l}\text { Grupo de } \\
\text { aeropuertos } \\
\text { internacionales }\end{array}$ & DEA \\
\hline $\begin{array}{l}\text { Domney et al, } \\
2005\end{array}$ & $\begin{array}{l}\text { No hay relación estadísticamente significativa con la } \\
\text { eficiencia }\end{array}$ & $\begin{array}{l}\text { Australia y Nue- } \\
\text { va Zelandia }\end{array}$ & DEA \\
\hline $\begin{array}{l}\text { Vogel \& Gra- } \\
\text { ham, 2006 }\end{array}$ & $\begin{array}{l}\text { Los aeropuertos parcial y totalmente privatizados son } \\
\text { más eficientes }\end{array}$ & Europa & $\begin{array}{l}\text { DEA+ } \\
\text { Análisis } \\
\text { de ratios }\end{array}$ \\
\hline $\begin{array}{l}\text { Malighetti, } \\
\text { P.; Paleari, S; } \\
\text { Redondi, R, } \\
\text { 2007 }\end{array}$ & $\begin{array}{l}\text { Los aeropuertos privados operan de manera más efi- } \\
\text { ciente la gestión del tráfico de aeronaves }\end{array}$ & $\begin{array}{l}\text { DEA+ } \\
\text { Índice de } \\
\text { Italia }\end{array}$ & $\begin{array}{l}\text { vidad de } \\
\text { valmquist }\end{array}$ \\
\hline
\end{tabular}

Revista Ingenierías Universidad de Medellín 


\begin{tabular}{|c|c|c|c|}
\hline Artículo & Principal conclusión & Caso de estudio & Método \\
\hline $\begin{array}{l}\text { Barros \& } \\
\text { Dieke, } 2007\end{array}$ & Los aeropuertos privados son más eficientes & Italia & \\
\hline $\begin{array}{l}\text { Fung et al, } \\
2008\end{array}$ & $\begin{array}{l}\text { Los aeropuertos públicos que cotizan en bolsa son } \\
\text { más eficientes que aquellos públicos que no cotizan en } \\
\text { bolsa }\end{array}$ & China & $\begin{array}{l}\text { DEA+ } \\
\text { Índice de } \\
\text { producti- } \\
\text { vidad de } \\
\text { Malmquist }\end{array}$ \\
\hline $\begin{array}{l}\text { Yiu, M.K; } \\
\text { Hong, K; Hui, } \\
\text { Y; Law, J.S, } \\
2008\end{array}$ & Los aeropuertos públicos son menos eficientes & China & $\begin{array}{l}\text { DEA+ } \\
\text { Índice de } \\
\text { producti- } \\
\text { vidad de } \\
\text { Malmquist }\end{array}$ \\
\hline $\begin{array}{l}\text { Yuen, A; } \\
\text { Zhang, A, } \\
2009\end{array}$ & Los aeropuertos públicos son menos eficientes & China & $\begin{array}{l}\text { DEA+ } \\
\text { Índice de } \\
\text { producti- } \\
\text { vidad de } \\
\text { Malmquist }\end{array}$ \\
\hline $\begin{array}{l}\text { Curi, C; } \\
\text { Gitto, S; Man- } \\
\text { cuso, P, } 2010\end{array}$ & $\begin{array}{l}\text { Los aeropuertos públicos son, en términos generales, } \\
\text { más eficientes }\end{array}$ & Italia & \begin{tabular}{|l|} 
DEA + \\
procedi- \\
miento \\
Simar y \\
Wilson \\
\end{tabular} \\
\hline $\begin{array}{l}\text { Assaf, A.G; } \\
\text { Gillen, D, } \\
2012\end{array}$ & $\begin{array}{l}\text { Los aeropuertos cuya gestión es privada son más } \\
\text { eficientes }\end{array}$ & $\begin{array}{l}\text { Grupo de } \\
\text { aeropuertos } \\
\text { internacionales }\end{array}$ & $\begin{array}{l}\text { DEA+ } \\
\text { Modelo } \\
\text { esto- } \\
\text { cástico } \\
\text { Bayesiano }\end{array}$ \\
\hline $\begin{array}{l}\text { Perelman, S; } \\
\text { Serebrisky, T, } \\
2012\end{array}$ & $\begin{array}{l}\text { Los aeropuertos privados son, en términos generales, } \\
\text { más eficientes }\end{array}$ & América Latina & $\begin{array}{l}\text { DEA+ } \\
\text { Índice de } \\
\text { producti- } \\
\text { vidad de } \\
\text { Malmquist }\end{array}$ \\
\hline
\end{tabular}

Fuente: Elaboración propia

Uno de los primeros trabajos que se conocen es el de Parker [28] quien investiga el impacto de la privatización en una muestra de 22 aeropuertos británicos y halla que no existe ningún impacto sobre la eficiencia. Años más tarde, Oum et al. [29] consideran que la estructura de propiedad de un aeropuerto no parece tener ningún efecto estadísticamente significativo sobre el desempeño de su productividad.

En el Reino Unido, Holvad y Graham [30] concluyen que el tipo de propiedad no tiene efecto sobre la eficiencia. En Australia y Nueva Zelanda, el estudio es llevado 
a cabo por Domney, Wilson and Chen [31] cuyos resultados indican que la privatización es asociada negativamente con la rentabilidad y, a su vez, no es estadísticamente significativa su asociación con la eficiencia.

En relación con estudios sobre grupos de aeropuertos de distintos países, Vogel y Graham [32] analizan diversos aeropuertos europeos, y llegan a la conclusión de que los aeropuertos parcial y totalmente privatizados presentan mejor desempeño, es decir, son más eficientes.

Para [33] los aeropuertos privados son más eficientes en lo que tiene que ver con la gestión de los movimientos de aeronaves en comparación con los aeropuertos públicos. Barros y Dieke [34], utilizando como caso de estudio un grupo de aeropuertos italianos, hallan que los aeropuertos privados operan de forman más eficiente que los aeropuertos públicos. En China [12] evalúan 25 aeropuertos y sus resultados muestran que los aeropuertos operados por el ente público fueron menos eficientes que los gestionados por otro tipo de propiedad. De nuevo, [35] analizan los aeropuertos de China y encuentran que los aeropuertos operados públicamente fueron más eficientes.

Curi et al. [36] encuentran en su estudio para 36 aeropuertos italianos que los aeropuertos cuya propiedad está mayoritariamente en manos de fondos públicos son en promedio más eficientes mientras que la presencia de un $H u b$ (aeropuerto que opera como centro principal de conexión de una aerolínea) es una fuente de ineficiencia.

Por último, en un trabajo más reciente [2] hallan que la mayoría de los aeropuertos eficientes son aquellos cuyo tipo de gestión es privada. De la misma forma se confirma en el estudio de [20] cuyos resultados indican que, para el caso de 21 aeropuertos latinoamericanos, los aeropuertos operados de forma privada se desempeñan mejor, en promedio, que los aeropuertos públicos.

\section{CONCLUSIONES}

La desregulación de la industria aérea mundial ha aumentado las demandas de servicios aeroportuarios, así como también la demanda de procesos más rápidos y más eficientes de aeronaves, pasajeros, carga y equipaje. Las aerolíneas precisan ubicar y expandir sus operaciones en aeropuertos eficientes de manera que logren reducir sus costos y mejorar la calidad de sus servicios. Este movimiento de liberalización también ha dado como resultado el crecimiento de una tendencia internacional de comercializar y privatizar aeropuertos.

Dos aspectos clave en el transporte aéreo parecen haber fortalecido la transformación general hacia la privatización de los aeropuertos. En primer lugar, a pesar 
de algunas incertidumbres relacionadas con el crecimiento del tráfico en los últimos años, todavía se espera un crecimiento a largo plazo y la mayoría de los aeropuertos no tienen la capacidad para hacer frente a esto [6]. Una nueva infraestructura necesita ser financiada y parece que hay cada vez más presión sobre la financiación del sector público, que era la principal fuente tradicional de financiación. En segundo lugar, muchos aeropuertos han demostrado a lo largo de los años que son capaces de ser operados como negocios comerciales y autosuficientes, en particular en respuesta a una industria aérea más exigente, desregulada y competitiva [6]. Así, el paradigma de servicio público en el que se ve a un aeropuerto como prestador de dicho servicio para satisfacer las necesidades de las compañías aéreas y los pasajeros, se ha sustituido por un paradigma comercial que considera un aeropuerto como una empresa de negocios.

En el nuevo escenario del transporte aéreo, conseguir mejores niveles de eficiencia y productividad son factores clave para permanecer en el mercado. Pero como se ha reseñado, encontrar los factores que favorezcan o desfavorezcan el logro de la eficiencia, no es asunto sencillo. Uno de estos factores que ha llamado la atención en la literatura académica ha sido el tipo de propiedad y/o gestión de las infraestructuras aeroportuarias.

El presente trabajo permite concluir que es bastante difícil realizar inferencias en términos generales, ya que los estudios reseñados llegan a conclusiones disímiles. Sin embargo, es posible apreciar una percepción generalizada en las conclusiones de los estudios y es que las empresas privadas tienen mayores posibilidades de un funcionamiento más eficiente, pero sin desconocer que estas requieren incentivos tendentes a la maximización de beneficios mediante aumento de precios, lo cual hace de la regulación un aspecto importante para aminorar aspectos negativos, pero sin desestimular los aspectos positivos [25].

Pese a todo lo mencionado previamente, la participación del sector privado en el desarrollo y gestión de los aeropuertos seguirá creciendo bajo el supuesto de posibilidad de expansión y renovación para atender a los estándares de calidad internacional. No obstante, este avance en la privatización seguirá dependiendo de los incentivos y del tipo de regulación.

\section{REFERENCIAS}

[1] E. Van De Vijver, B. Derudder y F. Witlox, «Exploring causality in trade and air passenger travel relationships: The case of Asia-Pacific, 1980-2010. J.», ournal of Transport Geography , vol. 34, pp. 142-150, 2014.

[2] A. Assaf y D. Gillen, «Measuring th joint impact of governance from and economic airport efficiency», European Journal of Operational Research, vol. 220, pp. 187-198, 2012. 
[3] M. Bazargan y B. Vasigh, «Size versus efficiency: a case study of US commercial airports», Journal of Air Transport Management, vol. 9, pp. 187-193, 2003.

[4] A. Graham, "Airport benchmarking: A review of the current situation», Benchmarking: An International Journal, vol. 12, n. ${ }^{\circ}$ 2, pp. 99-111, 2005.

[5] A. Graham, Managing airports: An international perspective (3rd Ed.), Oxford: ButterworthHeinemann, 2008.

[6] A. Graham, «Graham, A. (2011). The objectives and outcomes of airport privatisation», Research in Transportation Business \& Management, vol. 1, pp. 3-14, 2011.

[7] J. Martín y C. Román, «Political opportunists and Mavericks? A typology of Spanish airports», International Journal of Transport Economics, vol. 34, n. ${ }^{\circ}$ 2, pp. 245-269, 2007.

[8] F. Tapiador, A. Mateors y J. Martí-Henneberg, «The geographical efficiency of Spain's regional airports: a quantitative analysis», Journal of Air Transport Management, vol. 14, n. ${ }^{\circ}$, pp. 205-212, 2008.

[9] P. Coto-Millán, P. Casares-Hontañón, V. Inglada, M. Agûeros, M. Pesquera y A. Badiola, «Small is beautiful? The impact of economic crisis, low cost carriers, and size on efficiency inSpanish airports (2009-2011)», Journal of Air Transport Management, vol. 40, pp. 34-41, 2014.

[10] S. Susuki, P. Nijkamp, P. Rietveld y E. Pels, «A distance friction minimization approach in data envelopment analysis: A comparative study on airport efficiency», European Journal of Operational Research, vol. 207, pp. 1104-1115., 2010.

[11] N. Adler, V. Liebert y E. Yazhemsky, «Benchmarking airports from a managerial perspective», Omega, vol. 41, n. ${ }^{\circ}$, pp. 442-458, 2013.

[12] M. Yiu, K. Hong, Y. Hui y J. Law, «Productivity changes in Chinese airports 1995-2004», Transportation Research Part E, vol. 44, pp. 521-542, 2008.

[13] A. Chi-Lok y A. Zhang, «Effects of competition and policy changes on Chinese airport productivity: An empirical investigation», Journal of Air Transport Management, vol. 15, pp. 166-174, 2009.

[14] C. Wing y . M. Yiu, «Estimating technical efficiencies of airports in the Greater China: stochastic output distance function method vs. data envelopment analysis method», International Journal of Shipping and Transport Logistics, vol. 2, n. ${ }^{\circ}$ 3, pp. 284-299, 2010.

[15] M. Yiu y C. Wing, «Note on the productivity convergence of airports in China», Pacific Economic Review, vol. 16, n. ${ }^{\circ}$ 1, pp. 120-133., 2011.

[16] Y. Y. M. a. C. P. 17. Chang, «Evaluating the performance of Chinese airports», Journal of Air Transport Management, vol. 31, n. ${ }^{\circ}$ 6, pp. 19-21, 2012.

[17] P. Wanke, «Efficiency of Brazil's airports: evidences from bootstrapped DEA and FDH estimates», Journal of Air Transport Management , vol. 23, pp. 47-53, 2012. 
[18] L. Flor y B. de la Torre, «Medición no paramétrica de eficiencia y productividad total de los factores: el caso de los aeropuertos regionales de Perú», Revista de Regulación en Infraestructura de Transporte, vol. 1, pp. 100-115, 2008.

[19] C. Barros, «Airports in Argentina: Technical efficiency in the context of an economic crisis», Journal of Air Transport Management, vol. 14, pp. 315-319.

[20] S. Perelman y T. Serebrisky, «Measuring the technical efficiency of airports in Latin America», Utilities Policy, vol. 22, pp. 1-7, 2012.

[21] A. Charnes, W. Cooper y E. Rhodes, «Measuring the efficiency of decision making units», European Journal of Operational Research, vol. 2, pp. 429-444, 1978.

[22] J. Sarkis y S. Talluri, «An analysis of the operational efficiency of major airports in the United States», Journal of Operations Management, vol. 18, n. ${ }^{\circ}$ 3, pp. 335-351, 2004.

[23] D. Gillen y A. Lall, «Developing measures of airport productivity and performance: An application of data envelopment analysis», Transportation Research Part E, 33(4), 261-273, vol. 33, n. ${ }^{\circ}$, pp. 261-273, 1997.

[24] L. Ling y C. Hong, «Operational performance evaluation of international major airports: An application of data envelopment analysis», Journal of Air Transport Management, vol. 12, pp. 342-351, 2006.

[25] M. Izkue Rodríguez, «Evaluación de modelos de gestión y financiación aeroportuaria», Universitat Politècnica de Catalunya, Barcelona, 2011.

[26] F. Salazar de la Cruz, Introducción a la gestión económica de aeropuertos, Madrid: Fundación AENA, 2006.

[27] C. Oliveira Cruz y R. Cunha Marques, «Contribution to the study of PPP arrangements in airport development, management and operation», Transport Policy, vol. 18, pp. 392-400, 2011.

[28] D. Parker, «The performance of BBA before and after privatization», Journal of Transport Economics and Policy, vol. 33, n. ${ }^{\circ}$ 2, pp. 133-146, 1999.

[29] T. Oum, C. Yu y X. Fu, «A comparative analysis of productivity performance of the world's major airports: Summary report of the ATRS global airport benchmarking research report», Journal of Air Transport Management, vol. 9, n. ${ }^{\circ}$ 5, pp. 285-297, 2003.

[30] T. Holvad y A. Graham, «Efficiency measurement for UK Airports: An application of data envelopment analysis», The Empirical Economics Letters, vol. 3, n. ${ }^{\circ}$ 1, pp. 31-39, 2004.

[31] M. Domney, H. Wilson y E. Chen, «Natural monopoly privatisation under different regulatory regimes», International Journal of Public Sector Management, vol. 18, n. 3, pp. 274-292, 2005 .

[32] H. Vogel y A. Graham, "A comparison of alternative airport performance measurement techniques: A European case study,» Journal of Airport Management, vol. 1, n. ${ }^{\circ}$ 1, pp. 59-74, 2006. 
[33] P. Malighetti, S. Paleari y R. Redondi, «An empirical investigation on the efficiency, capacity and ownership of Italian airports», Rivista di Politica Economica, vol. 97, pp. 57-188., 2007.

[34] C. Barros y P. U. Dieke, «Barros, C. P., \& Dieke, P. U. C. (2007). Performance evaluation of Italian airports: A data envelopment analysis», Journal of Air Transport Management, vol. 13, n. ${ }^{\circ}$, pp. 184-191., 2007.

[35] A. Yuen y A. Zhang, «Effects of competition and policy changes on Chinese airport productivity: An empirical investigation», Journal of Ari Transport Mangement, vol. 15, pp. 166-174, 2009.

[36] C. Curir, S. Gitto y P. Mancuso, «The Italian airport industry in trasnition: a performance analysis», Journal of Air Transport Management, vol. 16, pp. 218-221, 2010.

[37] T. Oum, N. Adler y C. Yu, «Privatization, corporatization, ownership forms and their effects on the performance of the world's major airports», Journal of Air Transport Management, vol. 12, pp. 109-121, 2006. 